\title{
Diferencias en la flora herbácea y arbustiva entre claros y áreas bajo dosel en un bosque de Nothofagus pumilio en Argentina
}

\author{
Differences in the herb and shrub flora growing under canopy gaps and under closed \\ canopies in a Nothofagus pumilio forest of Argentina
}

M. ANGÉLICA DAMASCOS ${ }^{1,2} \&$ EDUARDO H. RAPOPORT ${ }^{1,3}$

${ }^{1}$ Departamento de Ecología, Universidad Nacional del Comahue, 8400 Bariloche, Argentina;
e-mail: pmad@iris.ufscar.br ${ }^{2}$; rapoport@cab.cnea.gov.ar ${ }^{3}$

\section{RESUMEN}

Se comparó la distribución de las hierbas y arbustos bajo el dosel y en dos tamaños de claros del bosque deciduo de Nothofagus pumilio de Argentina. La riqueza total y promedio de especies por cuadrado fueron similares bajo el dosel y en los claros pequeños (diámetro mayor $<5 \mathrm{~m}$ ) y medianos (diámetro mayor entre 5-10 m). Las especies generalistas fueron las más numerosas (46\%), mientras que el $19 \%$ correspondió a especies heliófobas y el $15 \%$ a heliófilas obligadas y facultativas. La ausencia de diferencias en la riqueza de especies entre los ambientes comparados puede ser atribuida a: (1) la alta frecuencia de especies generalistas, (2) el bajo efecto de la formación de los claros en la distribución de las especies heliófobas, (3) la persistencia de especies heliófobas en los claros, principalmente pequeños, (4) la invasión de los claros por especies heliófilas obligadas. La heterogeneidad del ambiente lumínico bajo el dosel y el efecto sobre las condiciones microclimáticas de los claros de los árboles marginales y los arbustos, son posibles causas de los patrones encontrados. Aunque con variaciones entre distintas formas de vida, las especies del bosque estudiado son probablemente tolerantes al bajo nivel de alteración generado por la dinámica natural del bosque.

Palabras clave: especies heliófobas, especies heliófilas, especies generalistas, dinámica de claros, riqueza de especies, sotobosque.

\begin{abstract}
The distribution of herbs and shrubs under closed canopies and in two canopy gaps of different size was compared in a Nothofagus pumilio deciduous forest of Argentina. The total and the average species richness per plot were similar between the areas under the canopy and in small (maximum diameter $<5 \mathrm{~m}$ ) and medium size gaps (maximum diameter $5-10 \mathrm{~m})$. Generalist species were the most numerous $(46 \%)$, while obligate and facultatively shade-tolerant (heliophobous) and intolerant (heliophilous) species accounted for 19 and $15 \%$ of the species, respectively. The absence of differences in species richness may be attributed to: (1) high frequency of generalist species (2) low effect of gap formation on shade-tolerant species distribution, (3) persistence of facultatively shade-tolerant species in small gaps, (4) gap invasion by obligate shade-intolerant species. The heterogeneity of light environment beneath the canopy and the effects on gap microclimate of edge trees and gap shrubs are possible causes of these patterns. Although with variations between the different biological groups, forest species are probably tolerant to the low level of disturbance generated by the natural forest dynamics.
\end{abstract}

Key words: canopy gaps, generalist species, shade-tolerant species, shade-intolerant species, species richness, understory.

\section{INTRODUCCIÓN}

La formación de claros en el dosel del bosque puede determinar profundos cambios en las condiciones de luz y modificar la temperatura, humedad y riqueza de nutrientes del suelo (Denslow 1980, 1987, Brokaw 1985). Estas diferencias afectan la regeneración de las especies vegetales, pudiéndose distinguir entre árboles tolerantes a la sombra, que sólo regeneran bajo el dosel, y los intolerantes, que requieren de las condiciones físicas reinantes en los claros (Whitmore 1989).
Mientras diversos estudios de las comunidades forestales han examinado el rol de los claros en la regeneración de las especies arbóreas (Bazzaz 1979, Canham 1989, Shupp et al. 1989, Veblen 1989, Rusch 1992, Arévalo \& Fernández-Palacios 1998), las hierbas y arbustos han recibido menos atención. Popma et al. (1992) incluyeron a las plantas arbustivas en la clasificación de las especies leñosas de bosques tropicales según su preferencia lumínica y Collins et al. (1985) clasificaron en forma análoga a las hierbas de bosques templados del Hemisferio Norte. 
El conocimiento de la distribución de las especies de plantas en relación a las diferencias de luz dentro del bosque, es útil para predecir futuros cambios debidos a la fragmentación o manejo del mismo y planificar la conservación de la diversidad local. Sin embargo, esta información es sumamente escasa para los bosques templados de Sudamérica. Para el bosque de Austrocedrus chilensis (Don) Florin et Boutleje del Sur de Argentina se clasificaron las especies herbáceas y leñosas en relación a su preferencia lumínica y se mostraron las diferencias en morfología y crecimiento entre claros y áreas bajo dosel (Damascos 1998). En el presente trabajo discutimos las consecuencias del régimen lumínico en el interior del bosque deciduo de Nothofagus pumilio (P. et E.) Krasser (Fagaceae) sobre la riqueza de hierbas y arbustos y la tolerancia de estas especies a distintos niveles de luz. Este bosque se encuentra poco alterado pero está siendo afectado por actividades turísticas crecientes. Se analiza si los claros originados por la dinámica natural del bosque son colonizados diferencialmente por distintas formas de vida.

\section{MATERIALES Y MÉTODOS}

\section{Área de estudio}

Se estudiaron los bosques de $N$. pumilio ubicados a $1.300 \mathrm{~m}$ de altitud en la ladera norte del cerro Otto, en los alrededores de San Carlos de Bariloche $\left(41^{\circ} 08^{\prime} \mathrm{S}, 71^{\circ} 18^{\prime} \mathrm{O}\right)$. En la zona la precipitación media varía entre 1.200 y $1.600 \mathrm{~mm}$ anuales y la temperatura media anual es de $8,8{ }^{\circ} \mathrm{C}$, registrada esta última a una altitud de $700 \mathrm{~m}$ en el Aeropuerto de Bariloche. De todos modos, en el sitio de muestreo, aplicando la regla bioclimática de Hopkins (descenso de $0,6^{\circ} \mathrm{C}$ por cada incremento de $100 \mathrm{~m}$ de altitud), se puede estimar en $5,2^{\circ} \mathrm{C}$ la temperatura media anual del bosque de Nothofagus pumilio. Las áreas estudiadas tenían un bajo nivel de alteración, con escasas a nulas evidencias de corte de plantas leñosas y sin ganado.

\section{Muestreo}

El estudio de la distribución de especies en claros y áreas bajo dosel se realizó en 15 parcelas de 20 x 20 m elegidas al azar, en cuyo interior se ubicaron, también al azar, 10 cuadrados de $1 \times 1 \mathrm{~m}$. Para recabar información microclimática y analizar la preferencia de las plantas por claros de diferentes tamaños, se usó una parcela adicional de $50 \times 50 \mathrm{~m}$, en la que se ubicaron al azar 50 cuadrados de $2 \times 2 \mathrm{~m}$.
En cada cuadrado de las parcelas de $20 \times 20 \mathrm{~m}$ y de la parcela de 50 x 50 m se registró la presencia o ausencia (claro) de cobertura arbórea y las especies herbáceas y arbustivas presentes. En la parcela de 50 × $50 \mathrm{~m}$ se indicó además si el cuadrado estaba ubicado en un claro pequeño, si su diámetro mayor estimado era $<5 \mathrm{~m}$, o mediano, si estaba entre 5 y $10 \mathrm{~m}$.

\section{Mediciones}

En la parcela de 50 x $50 \mathrm{~m}$ se obtuvieron registros de temperatura del suelo y se tomaron muestras de suelo superficial para determinar su contenido de humedad. La información fue tomada a mediados de febrero de 1996 entre las 13:00 y las 17:00 $\mathrm{h}$, en un día con cielo descubierto, $20{ }^{\circ} \mathrm{C}$ de temperatura y $30 \%$ de humedad relativa. Con un termómetro digital se realizaron en cada cuadrado tres mediciones consecutivas de temperatura del suelo libre de hojarasca a $10 \mathrm{~cm}$ de profundidad, las que fueron promediadas obteniéndose un único valor por cuadrado. A igual profundidad, se tomó una muestra de suelo en el centro de cada cuadrado para determinación de su contenido de humedad por el método gravimétrico.

\section{Análisis de los datos}

La información recabada en las 15 parcelas fue usada para calcular la frecuencia de cada especie herbácea y arbustiva bajo el dosel y en los claros. Debido al reducido número de especies de enredaderas, estas fueron consideradas dentro del grupo de las hierbas. Se identificaron, en primer lugar, las especies exclusivas de los claros (heliófilas obligadas) y las de áreas bajo el dosel (heliófobas obligadas). Para las especies que estaban presentes tanto en claros como bajo dosel, se determinó su preferencia por una de estas condiciones comparando su frecuencia mediante la prueba t de Student para muestras apareadas (Zar 1999). De esta forma, las hierbas y arbustos del bosque fueron clasificados como: (1) heliófilas facultativas, aquellas especies con una frecuencia significativamente mayor $(\mathrm{P}<0,05)$ en $\operatorname{los}$ claros; (2) heliófobas facultativas, las plantas que poseían una frecuencia significativamente mayor ( $\mathrm{P}<0,05)$ bajo el dosel y, (3) generalistas, especies cuya frecuencia no difirió estadísticamente entre los claros y las áreas bajo el dosel. Las especies presentes en menos de tres parcelas (raras) fueron excluidas de este análisis.

La información sobre la presencia de cada especie en claros pequeños y medianos se obtuvo en la 
parcela de 50 × $50 \mathrm{~m}$. Con estos datos se analizó la diferencia en la riqueza de especies de los diferentes ambientes del bosque, seleccionándose al azar 10 cuadrados ubicados bajo el dosel, 10 en claros pequeños y 10 en claros medianos. El número de especies en cada micro-hábitat se comparó mediante un análisis de varianza (Zar 1999) con dos factores: ambiente (bajo el dosel, claros pequeños y claros medianos) y forma de vida (hierbas y arbustos) y con 10 repeticiones por cada condición.

Los valores de temperatura y humedad del suelo superficial se compararon en forma separada entre los claros medianos, pequeños y las áreas ubicadas bajo dosel mediante análisis de varianza simple, usando el procedimiento de comparaciones múltiples de Student-Newman-Keuls, como una prueba a posteriori (Zar 1999).

\section{RESULTADOS}

La temperatura del suelo superficial fue significativamente menor $(\mathrm{P}<0,05)$ en las áreas bajo dosel (media $\pm \mathrm{DE}=10,6 \pm 0,9^{\circ} \mathrm{C}$ ) que en los claros pequeños $\left(15,2 \pm 1,0^{\circ} \mathrm{C}\right)$ y medianos $(21,4$ $\left.\pm 1,8^{\circ} \mathrm{C}\right)$. El contenido de humedad del suelo superficial bajo el dosel $(21,1 \pm 4,9 \%)$ fue superior $(\mathrm{P}<0,05)$ que en los claros pequeños $(7,1 \pm$ $3,1 \%)$ y medianos $(5,7 \pm 1,8 \%)$. Los dos tamaños de claros difirieron entre sí en la temperatura media del suelo superficial $(\mathrm{P}<0,05)$ pero no en su contenido de humedad.

Se identificaron en total 26 especies de plantas, 18 estaban presentes bajo el dosel, 16 en los claros pequeños y 14 en los claros medianos (Tabla 1). El número promedio de especies por cuadrado (Fig. 1) no difirió estadísticamente (análisis de varianza, Tabla 2) entre hierbas y arbustos, ni entre las áreas bajo el dosel, los claros pequeños y los medianos.

$\mathrm{Si}$ se excluye a las cinco especies presentes en menos de tres parcelas (especies raras, Tabla 1), los porcentajes de heliófilas, heliófobas y generalistas fueron de 15,19 y $46 \%$, respectivamente. Las especies generalistas resultaron ser las más numerosas tanto entre las hierbas como entre los arbustos, en los claros y en las áreas bajo el dosel (Fig. 2 y 3). No hay una separación completa de tipos de especies por ambiente, dado que en los claros, principalmente en los pequeños, persistían especies heliófobas facultativas (dos herbáceas y una arbustiva) y bajo el dosel se encontró también una especie heliófila facultativa (Tabla 1). Sólo dos especies herbáceas fueron exclusivas de las áreas bajo el dosel (Tabla 1).

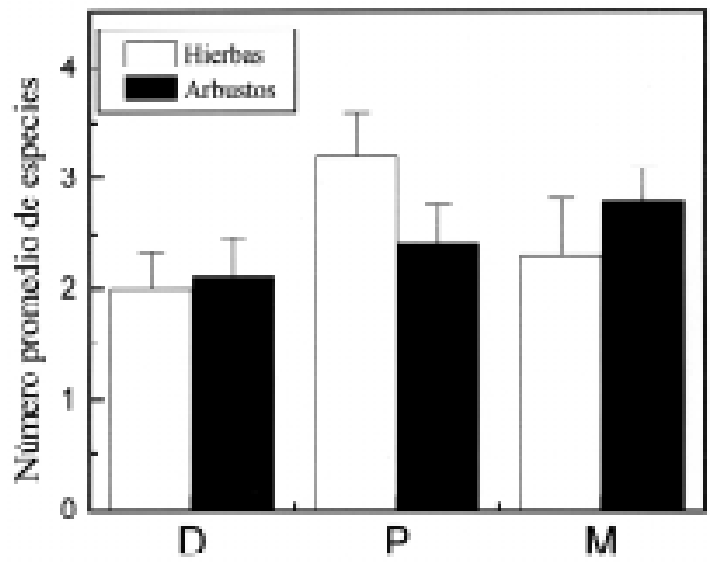

Fig. 1: Número (promedio \pm error standard) de especies de hierbas y arbustos presentes bajo el dosel (D) y en los claros pequeños (P, diámetro máximo $<5 \mathrm{~m}$ ) y medianos ( $\mathrm{M}$, diámetro máximo entre 5-10 m) del bosque de N. pumilio.

Herbs and shrubs species number (average \pm standard error) present under the canopy (D) and in the small (P, maximum diameter $<5 \mathrm{~m}$ ) and medium $(\mathrm{M}$, maximum diameter between $5-10 \mathrm{~m}$ ) gaps size of the $N$. pumilio forest.

\section{DISCUSIÓN}

A excepción de la invasión de los claros por un grupo de hierbas heliófilas obligadas (Tabla 1), las diferencias físicas entre los claros y las áreas ubicadas bajo dosel del bosque de $N$. pumilio no se tradujeron en diferencias substanciales de la composición de especies (Tabla 1), ni de la riqueza promedio de hierbas y arbustos (Fig. 1). El número promedio de especies por parcela en los claros del bosque estudiado (Fig. 1) no fue diferente del encontrado en claros generados experimentalmente por Duncan et al. (1998) en bosques de Nothofagus fusca (Hook.f.) Oest. y N. menziesii (Hook.f.) Oest. de Nueva Zelandia (2,6 \pm 1,4 a 3,7 $\pm 1,4$ especies en diferentes substratos). Estos autores observaron, además, una correlación positiva entre el área del claro y su riqueza de especies. Esta relación no fue constatada en el bosque de $N$. pumilio, posiblemente debido al bajo nivel de alteración presente (escasa remoción del suelo y corte de la vegetación), pues en este bosque los claros estudiados derivaban principalmente de su dinámica natural, eran de poca extensión, y en consecuencia los gradientes físicos en su interior serían poco marcados. Además de las hierbas heliófilas, los claros fueron invadidos por sólo dos hierbas exóticas (Tabla 1), sugiriendo también un bajo nivel de alteración. Hay que considerar que en el presente bosque de Nothofagus, la presencia de dos especies exóticas 
frente a 24 nativas, incluyendo las "raras", no llega al $8 \%$ de la flora. En áreas circundantes al mismo, los valores oscilan entre el 10 y $20 \%$, llegando hasta el $80 \%$ a pocos cientos de metros en donde se encuentra la zona suburbana de Bariloche (Rapoport 1993). La baja "contamina- ción por especies" registrada es, de alguna manera, un buen índice de la rigurosidad climática del bosque estudiado por nosotros.

Las áreas ubicadas bajo el dosel y los claros no difirieron en su riqueza de especies principalmente porque la mayor parte de estas fueron

TABLA 1

Especies del bosque de Nothofagus pumilio clasificadas según su tolerancia lumínica. Generalistas: especies cuya frecuencia no difiere entre las áreas sombreadas y los claros, Heliófobas obligadas: especies presentes sólo en áreas bajo el dosel, Heliófobas facultativas: especies con frecuencia significativamente mayor $(\mathrm{P}<0,05)$ en áreas bajo el dosel, Heliófilas obligadas: especies sólo presentes en los claros, Heliófilas facultativas: especies con frecuencia significativamente mayor $(\mathrm{P}<0,05)$ en los claros. Hábitats ocupados: (D) áreas bajo el dosel; (P) claro pequeño (diámetro mayor < $5 \mathrm{~m}$ ); (M) claro mediano (diámetro mayor entre 5-10 m); (\#) indica especie exótica

Species of the Nothofagus pumilio forest grouped according their light tolerance. Generalist: species with no significant differences in their frequency between the areas under the canopy and the gaps, Obligate shade-tolerant

species: species only present in the areas under the canopy, Facultative shade-tolerant species: species with frequency significatively greater $(\mathrm{P}<0.05)$ in areas under the canopy, Obligate shade-intolerant species: species only present in gaps, Facultative shade-intolerant species: species with frequency significatively greater $(\mathrm{P}<0.05)$ in gaps. Habitats used: (D) areas under the canopy; (P) small gap size (maximum diameter $<5$ m); (M) medium gap size (maximum diameter $5-10 \mathrm{~m}$ ); (\#) indicates exotic species

\begin{tabular}{|c|c|c|c|}
\hline Especie & Familia & Forma de vida & $\begin{array}{l}\text { Hábitat } \\
\text { ocupado }\end{array}$ \\
\hline
\end{tabular}

Generalistas

Acaena ovalifolia $\mathrm{R}$. et P.

Agrostis leptotricha Desv.

Berberis buxifolia Lam.

Cortaderia pilosa (Dürv.) Hackel

Galium hypocarpium (L.) Endlicher

Maytenus chubutensis (Speg.)

Lourt., O’Don. et Sleum.

Mutisia decurrens Cav.

Mutisia spinosa R. et P.

Ribes magellanicum Poir.

Schinus patagonicus (Phil.) Johnst.

Valeriana carnosa J. E. Sm.

Viola maculata Cav.

Rosaceae
Gramineae
Berberidaceae
Gramineae
Rubiaceae
Celastraceae
Compositae
Compositae
Saxifragaceae
Anacardiaceae
Valerianaceae
Violaceae

Geraniaceae

Papilionaceae

Geranium magellanicum Hook. f.

Vicia nigricans Hook et Arn.

Heliófobas facultativas

Alstroemeria aurantia Phil.

Berberis serrato-dentata Lechler

Osmorhiza chilensis $\mathrm{H}$. et A.

Heliófilas obligadas

Acaena pinnatifida R. et P.
Acaena splendens H. et A.
Phacelia secunda J. F. Gmel.

Heliófilas facultativas

Fragaria chiloensis (L.) Duch.
Amaryllidaceae

Berberidaceae

Umbelliferae

$\begin{array}{cc}\text { Hierba } & \text { DMP } \\ \text { Hierba } & \text { DMP } \\ \text { Arbusto } & \text { DMP } \\ \text { Hierba } & \text { DMP } \\ \text { Hierba } & \text { DP }\end{array}$

ArbustoDMP

Enredadera

Enredadera

Arbusto

Arbusto

Hierba

Hierba

DMP
DMP
DMP
DMP
DP
DP

Hierba

Enredadera

D

D

$\begin{array}{cc}\text { Hierba } & \text { DP } \\ \text { Arbusto } & \text { DMP } \\ \text { Hierba } & \text { DP }\end{array}$

Hierba $\quad M$

Hierba M

Hierba MP

Especies raras (presentes en menos de tres parcelas): Rumohra adiantiformis (Forst.) Ching (Polipodiaceae), Adenocaulon chilensis Less. (Compositae), Lathyrus magellanicum Lam. (Fabaceae), Rumex acetosella L. \# (Polygonaceae), Cerastium arvense L. \# (Caryophyllaceae) 


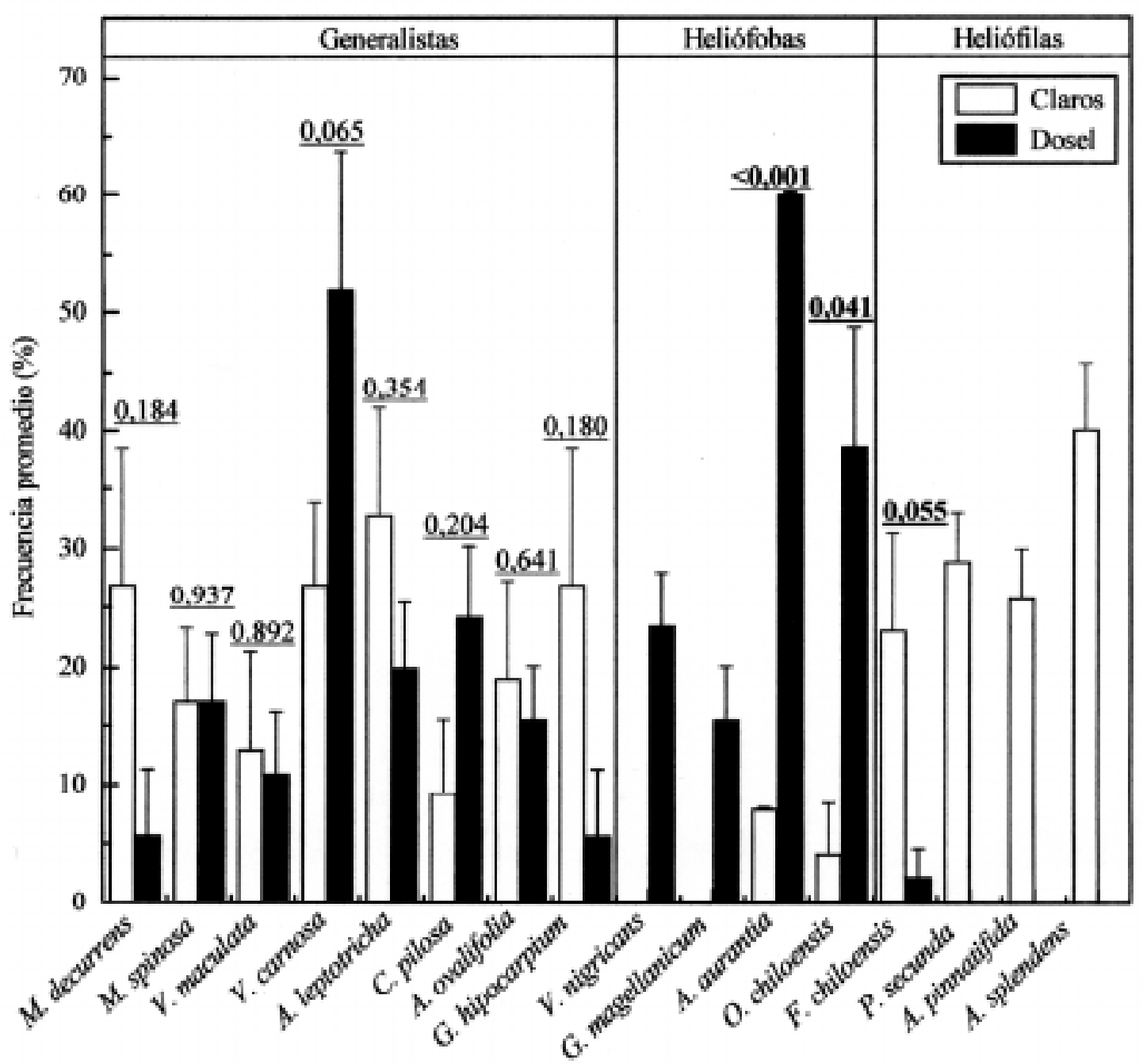

Fig. 2: Frecuencia (promedio \pm error standard) de las hierbas del bosque de N. pumilio en los claros y bajo el dosel. Los valores sobre las barras de frecuencia de cada especie indican la probabilidad de diferencias estadísticas (prueba t de Student para muestras pareadas, $\mathrm{P}<0,05$ ) entre ambas condiciones.

Frequency (mean \pm standard error) of the $N$. pumilio forest herbs in the gaps and under the canopy. The values above the frequency bars of each species indicates the probability of statistical significant differences (Student paired t-test, $\mathrm{P}<$ 0.05 ) between both studied conditions.

generalistas (Tabla 1). Arroyo et al. (1996) indicaron que en la mayoría de los bosques templados siempreverdes de Sudamérica, los arbustos tienden a ser más comunes en los claros y en los márgenes del bosque sin formar un estrato continuo. En el bosque estudiado esto no se cumple, debido a la dominancia de especies generalistas. Cuatro de los cinco arbustos presentes son generalistas (Tabla 1) mientras que el restante, Berberis serrato-dentata, es una especie umbrófila de poca altura (hasta $50 \mathrm{~cm}$ ) que es abundante bajo el dosel, pero persiste con baja frecuencia en los claros, probablemente debido al sombreado de los arbustos generalistas y de los árboles que bordean el claro. Los arbustos generalistas del bosque de $N$. pumilio (Ribes magellanicum, Berberis buxifolia, Maytenus chubutensis y Schinus patagonicus) están adaptados a un amplio rango de condiciones ambientales dentro de la región. Son especies de alta frecuencia tanto en los bosques ubicados a menor altitud, como los de Nothofagus dombeyi, presentes en áreas con 2.000$2.200 \mathrm{~mm}$ de precipitación promedio anual, como en los bosques de Austrocedrus chilensis extendidos sobre áreas más secas (1.000-1.500 mm), y también en matorrales secundarios (Ladio \& Damascos 2000). Esta dominancia de especies generalistas, fue también encontrada en el bosque de Austrocedrus chilensis de la misma región (Damascos 1998) y en claros de bosques templa- 


\section{TABLA 2}

Resumen de la tabla de análisis de varianza $(\mathrm{P}<0,05)$ que compara los efectos del ambiente (bajo el dosel, claros de tamaño pequeño, claros de tamaño mediano) y de la forma de vida (hierbas y arbustos) sobre la riqueza de especies. El valor de F corresponde al estadístico de Fisher

Summary of variance analysis table $(\mathrm{P}<0.05)$ comparing de effects of environment (areas under de canopy, small gap size, medium gap size) and the life form (herbs and woody species) on species richness. F values correspond to the Fisher's statistic

\begin{tabular}{|c|c|c|c|c|}
\hline Fuente de variación & Grados de libertad & Cuadrado medio & Valor de F & Valor de P \\
\hline Ambiente (A) & 2 & 2,9167 & 2,0038 & 0,1447 \\
\hline Tipo biológico (Tb) & 1 & 0,0667 & 0,0458 & 0,8313 \\
\hline $\mathrm{A} \times \mathrm{Tb}$ & 2 & 2,2167 & 1,5229 & 0,2273 \\
\hline Residual & 54 & 1,4556 & & \\
\hline
\end{tabular}

dos del Hemisferio Norte (Luken et al. 1997) y posiblemente derive del hecho que dichas especies poseen un conjunto de atributos que les permiten propagarse y persistir bajo el efecto de la alteración del bosque: capacidad de rebrote, multiplicación vegetativa por yemas gemíferas y producción de frutos carnosos que no se acumulan en

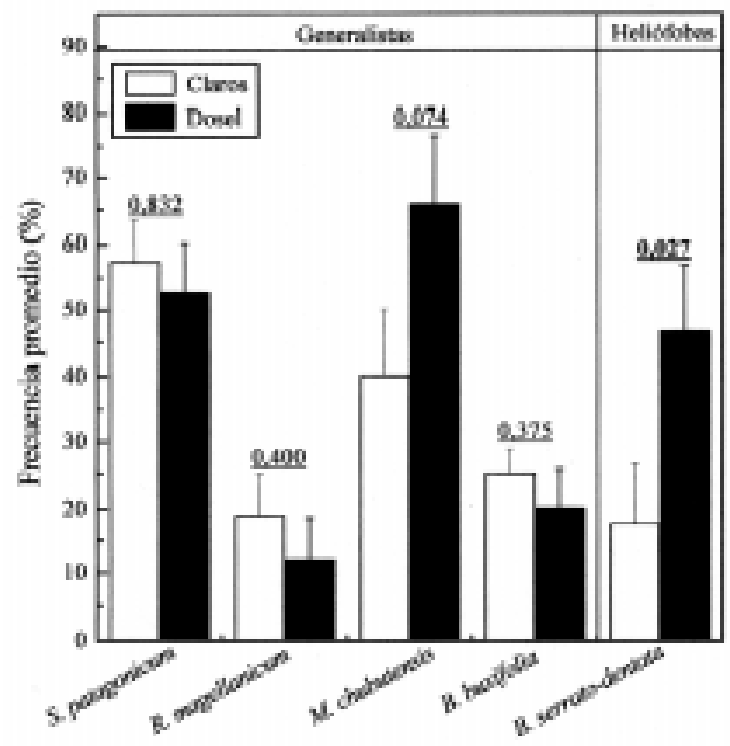

Fig. 3: Frecuencia (promedio \pm error standard) de las especies arbustivas del bosque de $N$. pumilio en los claros y bajo el dosel. Los valores sobre las barras de frecuencia de cada especie indican la probabilidad de diferencias estadísticas (prueba t de Student para muestras pareadas, $\mathrm{P}<0,05)$ entre ambas condiciones.

Frequency (mean \pm standard error) of the $N$. pumilio forest shrubs in the gaps and under the canopy. The values above the frequency bars of each species indicates the probability of statistical significant differences (Student paired t-test, $\mathrm{P}<0.05$ ) between both studied conditions. el banco permanente de semillas y germinan rápidamente (Damascos et al. 1999). Por otro lado, Berberis serrato-dentata, especie heliófoba del bosque de $N$. pumilio posee también los anteriores atributos.

La apertura de un claro supone la liberación de nuevos recursos, mayor acceso a luz, mayores gradientes físicos (Brokaw 1985), lo cual favorecería la instalación de nuevas especies, principalmente exóticas. Sin embargo el bosque de $N$. pumilio debido a su posición por encima de los $1.100 \mathrm{~m}$, tiene un relativo aislamiento, tanto físico como climático, que no favorece la invasión. Las especies generalistas con sus atributos de especies sucesionales aprovechan la variedad de condiciones físicas dentro del bosque. La persistencia en los claros, particularmente pequeños, de algunas plantas heliófobas facultativas (Tabla 1) fue también observada en bosques del hemisferio Norte (Collins et al. 1985). Dichas especies crecerían bajo la protección de los arbustos de mayor porte que crean dentro del claro condiciones microclimáticas por sombreado. Además como ya se indicó los claros, especialmente los pequeños, reciben la influencia de los árboles del borde.

En las áreas ubicadas bajo dosel la flora estaba integrada tanto por especies heliófobas y generalistas como por una especie heliófila (Tabla 1). La persistencia de los dos últimos grupos puede ser atribuida a la variedad de condiciones de luz debida a la heterogeneidad de la cobertura arbórea. El dosel de los bosques varía en composición específica, espesor y densidad del follaje arbóreo (Lieberman et al. 1989) y deja pasar "flecos de luz" que llegan hasta el piso del bosque (Aber \& Melillo 1991).

Estudiando la interacción entre los atributos del grupo de plantas, el tamaño del área alterada 
y la severidad de la alteración del bosque, Jonsson \& Esseen (1998) encontraron que la riqueza de especies fue afectada significativamente por la magnitud y la intensidad de la alteración y observaron que la tasa de recubrimiento de los claros fue mayor entre las briofitas debido a sus estrategias de regeneración. En el presente estudio, la distribución de especies analizadas abarcó sólo plantas vasculares, pero si se consideran las distintas formas de vida (hierbas, enredaderas y arbustos) se observa que las mayores diferencias, tanto por exclusión de especies heliófobas como por la invasión de plantas heliófilas, correspondió a las especies herbáceas. En general, las hierbas de las áreas sombreadas de los bosques son competidoras desiguales con respecto a los componentes estructurales dominantes de la comunidad y son relativamente intolerantes al estrés físico (Collins et al. 1985). Las hierbas heliófilas obligadas con mayor frecuencia en otras comunidades, se comportan como oportunistas en los bosques (Damascos 1998).

En resumen, debido especialmente al alto número de plantas generalistas en las distintas formas de vida y a sus atributos de regeneración, la formación de claros proveniente principalmente de la dinámica natural del bosque, no conduce a una diferenciación florística marcada con respecto a las áreas ubicadas bajo el dosel. Se registran diferencias en la abundancia de las especies (Fig. 2 y 3 ), lo que permite agruparlas según su preferencia a la luz (Tabla 1), invasión de los claros por unas pocas especies heliófilas obligadas, pero de un total de 18 especies nativas presentes bajo el dosel, sólo dos resultaron desplazadas de las áreas abiertas. Las distintas formas de vida de este bosque son probablemente tolerantes a los cambios en las condiciones físicas generadas por niveles bajos de alteración.

\section{AGRADECIMIENTOS}

Deseamos agradecer al Dr. Juan J. Armesto y a dos revisores anónimos por sus sugerencias y contribución a la calidad del manuscrito final.

\section{LITERATURA CITADA}

ABER J \& J MELILLO (1991) Terrestrial ecosystems. Saunders College Publishing, Philadelphia, Pennsylvania. 424 pp.

ARÉVALO JR \& JM FERNÁNDEZ-PALACIOS (1998) Treefall gap characteristics and regeneration in the laurel forest of Tenerife. Journal of Vegetation Science 9: 297-306.
ARROYO MK, L CAVIERES, A PEÑALOSA, M RIVEIROS \& AM FAGGI (1996) Relaciones fitogeográficas y patrones de riqueza de especies en la flora del bosque lluvioso templado de Sudamérica. En: Armesto JJ, C Villagrán \& MK Arroyo (eds) Ecología de los bosques nativos de Chile: 71-92. Editorial Universitaria, Santiago, Chile.

BAZZAZ F (1979) The physiological ecology of plant succession. Annual Review of Ecology and Systematics 10: 351-371.

BROKAW N (1985) Treefalls, regrowth, and community structure in tropical forests. En: Pickett A \& S White (eds) The ecology of natural disturbance and patch dynamics: 53-68. Academic Press, New York, New York.

CANHAM C (1989) Different responses to gaps among shade-tolerant tree species. Ecology 70: 548-550.

COLLINS B, K DUNNE \& S PICKETT (1985) Responses of forest herbs to canopy gaps. En: Pickett A \& S White (eds) The ecology of natural disturbance and patch dynamics: 218-234. Academic Press, New York, New York.

DAMASCOS M (1998) Morfología de los claros y las áreas sombreadas del bosque de Austrocedrus chilensis. Ecología Austral (Argentina) 8: 13-22.

DAMASCOS M, L GHERMANDI \& A LADIO (1999) About the persistence of the native species of a Patagonian Austrocedrus chilensis forest. International Journal of Ecology and Environmental Sciences 25: 21-35.

DENSLOW J (1980) Gap partitioning among tropical rainforest trees. Biotropica 12: 47-55.

DENSLOW J (1987) Tropical rainforest gaps and tree species diversity. Annual Review of Ecology and Systematics 18: 431-451.

DUNCAN RP, HL BUCKLEY, SC URLICH, GH STEWART \& J GERITZLEHNER (1998) Small-scale species richness in forest canopy gaps: the role of niche limitation versus the size of the species pool. Journal of Vegetation Science 9: 455-460.

JONSSON BG \& PA ESSEEN (1998) Plant colonization in small forest-floor patches: importance of plant group and disturbance traits. Ecography 21: 518-526.

LADIO AH \& MA DAMASCOS (2000) La invasión de especies exóticas y conservación de plantas nativas en baldíos suburbanos. Medio Ambiente (Chile) 13: 27-34.

LIEBERMAN M, D LIEBERMAN \& R PERALTA (1989) Forests are not just Swiss cheese: canopy stereogeometry of non-gaps in tropical forests. Ecology 70: 550-552.

LUKEN JO, LM KUDDES \& TC THOLEMEIR (1997) Response of understory species to gap formation and soil disturbance in Lonicera maackii thickets. Restoration Ecology 5: 229-235.

POPMA J, J BONGERS \& M WERGER (1992) Gapdependence and leaf characteristics of trees in a tropical lowland rain forest in México. Oikos 63: 207214.

RAPOPORT EH (1993) The process of colonization in small settlements and large cities. In: McDonnell, MJ \& S Pickett (eds) Humans as components of ecosystems: 190-201. Springer-Verlag, New York, New York. 
RUSCH V (1992) Principales limitantes para la regeneración de la lenga en la zona N.E. de su área de distribución. Variables ambientales en claros del bosque. Centro de Investigación y Extensión Forestal Andinopatagónico (CIEFAP), Publicación Técnica No. 8: 61-73.

SCHUPP E, H HOWE, C AUGSPURGER \& D LEVEY (1989) Arrival and survival in tropical treefall gaps. Ecology 70: 562-564.
VEBLEN T (1989) Tree regeneration responses to gaps along a transandean gradient. Ecology 77: 541-543. WHITMORE T (1989) Canopy gaps and the two major groups of forest trees. Ecology 70: 536-538.

ZAR JH (1999) Biostatistical analysis. Fourth edition. Prentice Hall, Englewood Cliffs, New Jersey. 663 pp.

Editor Asociado: J. Armesto

Recibido el 20 de mayo de 1997; aceptado el 25 de enero de 2002 